\title{
PulSync: The Heart Rate Variability as a Unique Fingerprint for the Alignment of Sensor Data Across Multiple Wearable Devices
}

\author{
Florian Wolling \& Kristof Van Laerhoven \\ Ubiquitous Computing \\ University of Siegen \\ Siegen, Germany \\ 0000-0002-4431-2378 \& 0000-0001-5296-5347
}

\author{
Pekka Siirtola \& Juha Röning \\ Biomimetics and Intelligent Systems Group \\ University of Oulu \\ Oulu, Finland \\ 0000-0002-5995-5421 \& 0000-0001-9993-8602
}

\begin{abstract}
Most off-the-shelf wearable devices do not provide reliable synchronization interfaces, causing multi-device sensing and machine learning approaches, e.g. for activity recognition, still to suffer from inaccurate clock sources and unmatched time. Instead of using active online synchronization techniques, such as those based on bidirectional wireless communication, we propose in this work to use the human heartbeat as a reference signal that is continuously and ubiquitously available throughout the entire body surface. We introduce PulSync, a novel approach that enables the alignment of sensor data across multiple devices utilizing the unique fingerprint-like character of the heart rate variability interval function. In an evaluation on a dataset from 25 subjects, we demonstrate the reliable alignment of independent ECG recordings with a mean accuracy of $-0.71 \pm 3.44$ samples, respectively $-2.86 \pm 11.43 \mathrm{~ms}$ at $250 \mathrm{~Hz}$ sampling rate.

Index Terms-wearable, synchronization, heart rate variability
\end{abstract}

\section{INTRODUCTION}

The proper alignment of independent time bases across multiple wearable devices, attached to various body locations, is a major challenge for a range of today's sensing tasks. Most off-the-shelf devices do not support hardware synchronization as it is complex and tends to exceed their small energy budget. For this reason, available research datasets contain often manually, and thus often inaccurately, matched sensor channels. Especially applications that apply advanced machine learning techniques on multi-modal sensor signals, such as activity recognition from wearable devices, are suffering from a poor alignment of coincident events as these inaccuracies inevitably result in a poor classification [1], [2].

Several approaches are aiming at the synchronization of nodes in wireless sensor networks (WSN) such as body-worn sensing systems. They can be divided into two fundamental categories. The first one, aiming for online synchronization, is usually network-based and relies on wireless technology such as Bluetooth [3]-[5]. The required protocols, however, result in an overhead in the already energy-intensive radio communication. The second one, aiming for offline synchronization, exploits spontaneous and sporadic events to align the recorded time series subsequently [2], [6]-[10]. Typical examples use external events such as motion patterns, to observe and identify simultaneously occurring events in the sensors' measurements to match the time bases. As these sensors are attached to the body, however, such a synchronization can suffer from soft tissue deformation and delays due to motion sequences and inertia of the body parts. Patterns in accelerometers' signals can therefore exhibit significant shifts: for a standing jump for instance, the arms' sensors produce a pattern first, followed by a delayed pattern in the sensors at the torso. In contrast to the above approaches, we propose to synchronise with the human heartbeat, a signal that is continuously and ubiquitously available throughout the entire body surface.

Different measurement principles enable the detection of the heartbeat, either by capturing the pulse wave that travels through the blood vessels, or by detecting the electric field, originated in the cardiac muscle, at the skin surface. For decades, electrocardiography (ECG) was exclusively present in clinical environments. In recent years, however, the progress in miniaturization and signal processing has enabled more convenient wearable devices to detect the tiny, pulsatile electric field changes at the body surface. It is predicted that most common wearables will soon have access to the vital sign through an on-board heart rate sensor [11], [12]. Inter-beat intervals for heart rate variability (HRV) analysis are commonly calculated from such signals, with the HRV interval function [13] being a unique, fingerprint-like signal exhibiting identical patterns at all body locations. ECG signals also have a high propagation velocity [14], so that the pulsatile signals show up nearly simultaneously throughout a person's body surface. ECG is thus an attractive reference for aligning time series across wearables, either as independent and offline recordings, or online and solely data-driven, based on the compressed information of the detected heartbeats' timestamps.

In this paper, we make the following contributions:

- We present PulSync, a novel approach for the data-driven alignment of sensors solely based on the human heartbeat.

- We propose to utilize the ubiquitously, continuously available vital sign and the derived location-independent, unique, and fingerprint-like heart rate variability (HRV) interval function to match the local times across multiple devices.

- Our evaluation demonstrates the method's general feasibility and an achieved alignment accuracy in the order of few ms for a publicly available dataset from 25 subjects [15]. 


\section{THEORETICAL BACKGROUND}

The following section first gives an overview of relevant online and offline synchronization methods for body-worn sensing systems. Subsequently, the origin of the heartbeat and its detection are described exemplary for ECG-related techniques. Finally, the heart rate variability and its unique, fingerprint-like characteristics are explained, which eventually enable the data-driven alignment of the signals.

\section{A. Present Synchronization Methods}

A large variety of methods and research approaches aims at the time synchronization in diverse applications with entirely different requirements on accuracy and precision as well as limits in costs and complexity. The established network time protocol (NTP) and the precision time protocol (PTP) are widely used in wired networks such as the Internet. Also the popular global positioning system (GPS) provides precise time information. In wireless sensor networks (WSN) and particularly in body area networks (BAN), with multiple bodyworn nodes, energy efficiency, and respectively the resulting battery life, is a critical parameter [11]. The surveys [16] and [17] provide a brief overview of common online synchronization techniques for WSN. Based on the short-range wireless standard Bluetooth, approaches for synchronized measurements in body sensor networks (BSN) achieved accuracies ranging from $3.5 \mathrm{~ms}$ [3] over $100 \mu \mathrm{s}$ using the sniff mode [4] to even $17.4 \mu \mathrm{s}$ utilizing the spark state mechanism which, however, is not supported by all devices [5].

The method of Bannach et al. [6] is not intended to compete with network-based online approaches. It rather aims at onbody applications of stand-alone devices without the capability of synchronization via wireless communication as it would exceed their energy budget. Instead, synchronization actions of the wearer, such as clapping, shaking, or jumping, are used as characteristic signatures to automatically align signal segments from different sensors. This content-based synchronization enables to reduce communication to a unidirectional channel or even an offline alignment of the recorded data. The quality of event spotting is essential for the accuracy of alignment, and for different sensing modalities specific spotting algorithms are required. However, the required synchronization performance largely depends on the target applications. According to the authors, an accuracy below $1 \mathrm{~s}$ is sufficient for the recognition of motion-related activities in daily life while a performance better than $0.1 \mathrm{~s}$ is considered as not required.

Bennett et al. [7], [8] present a data-driven offline synchronization technique that detects physical and cyber couplings between the interacting signals of multiple wearable sensors. Couplings are events that happen to the sensors at the same global time, irrespective of their local timestamps. A shortest path algorithm is then applied to determine those signal couplings that minimize the overall clock drift in the system.

Likewise, Wang et al. [2] present an advanced method that combines the single-time use of the energy-demanding NTP with the continuous identification and alignment of context markers. Similar to the previous signal couplings, these are physical actions that are known to have been detected simultaneously by multiple sensors. In contrast to predefined synchronization actions, context markers are incidentally performed throughout the entire recording and do not interrupt the experiment. The presented method reduced the synchronization error to $20 \mathrm{~ms}$ in comparison to the use of solely NTP with $250 \mathrm{~ms}$ or exclusively context markers with $1312 \mathrm{~ms}$.

Instead of explicit synchronization actions, signal couplings, or context markers, Hölzemann et al. [9] utilize the uniqueness of variations present in accelerometer signals from inertial measurement units (IMU) to align the independently recorded time series. The alignment, with an accuracy in the order of seconds, requires sufficient periods of resting with low variations as well as similarities and accordance in the simultaneous measurements at diverse locations such as head and wrist.

Ahmed et al. [10] present an approach that specifically aims at the multi-modal detection and analysis of cough events across multiple devices. Coughs are high energy events with a concise length of 0.3 to $0.7 \mathrm{~s}$ and simultaneously perceptible in audio and acceleration measurements. The alignment of these sporadic events, by means of a normalized cross-correlation, resulted in an average synchronization error of $46 \mathrm{~ms}$.

The entirely different approach of Li et al. [18] presents a time division multiple access (TDMA) protocol for mediumaccess control (MAC) which utilizes the rhythm of the heartbeat, instead of periodically broadcasted radio beacons, to schedule the time slots in star-topology wireless BSNs. Based on the assumption that body-worn devices have access to the wearer's vital signs, the "naturally synchronized" information are used to allocate time slots, to achieve a coarse synchronization without the need to enable the energy-consuming radio to receive periodic timing information.

\section{B. Human Heartbeat Detection}

The heart is the center of the human cardiovascular system and pumps the blood through the entire body. This physiological activity can be captured by means of different sensing modalities. Since each contraction of the cardiac muscle is initiated through action potentials, their accumulated electric field can be detected using electrocardiography (ECG). First experiments and the actual invention of traditional ECG can be traced back into the end of the $19^{t h}$ century. It is considered a gold standard in today's medicine, and an essential instrument to diagnose heart diseases and to reliably monitor heart rate. Each heartbeat generates a wave pattern with unique attributes, labeled with $\mathrm{P}, \mathrm{Q}, \mathrm{R}, \mathrm{S}$, and T, caused by superimposed stimuli in the cardiac muscle. To obtain the ECG signal's typical, wellknown contour, multiple wet electrodes are placed at specific locations on chest and extremities. Depending on the applied lead system, they detect the local electric potential differences along e.g. 12 leads, paired electrodes. The high reliability and accuracy of ECG originates from the sharp R peak of the characteristic QRS complex [19]. With its large amplitude, resulting in a high signal-to-noise ratio (SNR), the peak serves as a significant fiducial point that is less affected by noise and interference, and reliably detectable at most body locations. 

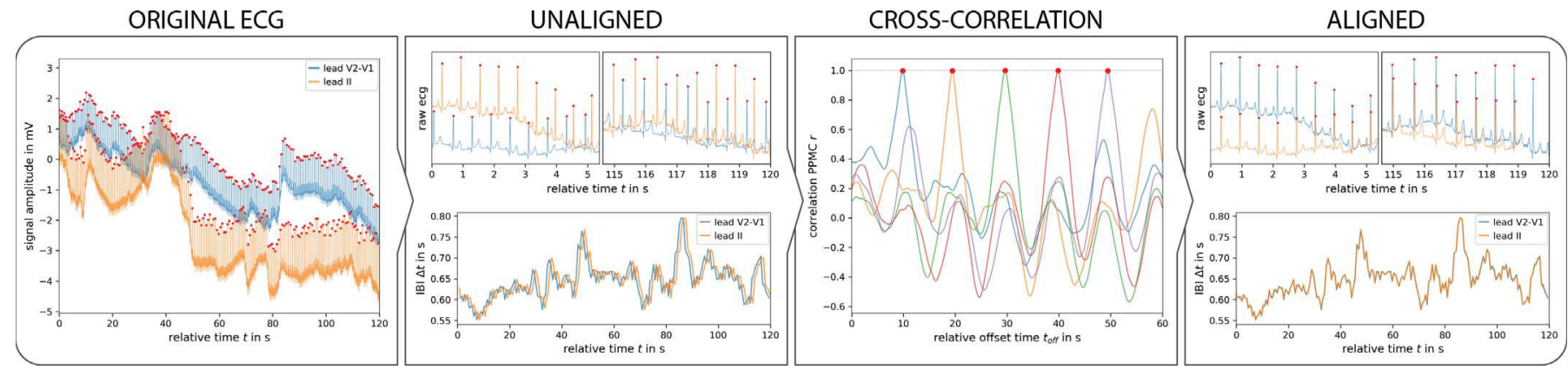

Fig. 1: PulSync processing pipeline for the alignment of independent wearables' time bases, applied on ECG measurements. Example from dataset 716 [15]: subject 23, Einthoven II (orange) and resembled V2-V1 (blue) leads. From left to right: 1) original ECG signals, labeled with precise fiducial points at the prominent R peaks (red); 2) derived HRV interval functions (bottom) from the original, unaligned time series (top); 3) identification of the positions with maximum accordance (red) and determination of the time series' relative alignment offset $\Delta$ using normalized cross-correlation; exemplary correlation of five $60 \mathrm{~s}$ segments $\alpha_{p}$ with their associated reference $\beta_{q} ; 4$ ) aligned interval functions (bottom) and ECG time series (top).

Therefore, ECG allows to determine the heart rate by either counting the number of peaks per unit time or by calculating the individual reciprocal of the R-R inter-beat interval (IBI) for an instantaneous measure [20].

Progress in signal processing and miniaturization are successively enabling advanced ECG techniques aiming at smaller and more convenient wearables for ambulant health care, longterm monitoring, and fitness tracking. Measured at a single spot [21], [22], such as the chest or even the wrist, the tiny electrical bio-potential differences are ranging from hundreds to only tens of $\mu \mathrm{V}$ [23] and require particularly sensitive analog front-ends with a very high input impedance beyond several G $\Omega$ [24]. The described setup inevitably results in a less robust signal with a low SNR and, depending on the lead orientation, the pulse can also show an inverse amplitude. Advanced filters nevertheless enable to extract and identify the prominent $\mathrm{R}$ peaks of the QRS complexes. An overview of common algorithms is provided by Köhler et al. [19].

To date, most wearable devices apply the optical measurement principle photoplethysmography (PPG) as it is cheap and easy to implement, however its accuracy still did not catch up with ECG [25]. PPG captures the pulse wave traveling through blood vessels at a low propagation velocity of about 5.2 to $14.6 \mathrm{~m} \mathrm{~s}^{-1}$ [26] and thus suffers from a strong location dependency and a varying pulse arrival time (PAT), depending on the distance from the heart. In contrast, the electrical ECG signal showed a lower-bound velocity of at least $250 \mathrm{~m} \mathrm{~s}^{-1}$ [14].

Consequently, the general feasibility of PulSync is evaluated on ECG signals as the prominent $\mathrm{R}$ peaks can be assumed to be immediately and simultaneously detectable throughout the entire body surface. However, future enhancements might enable to use other techniques such as the optical PPG.

\section{Heart Rate Variability}

The pointed ECG signal shows a pseudo-periodic run [13], influenced by physiological processes which modulate its intensity, amplitude, and pulse frequency [27]. The quasirandom modulation of the IBI is of particular interest as it contains frequency components which reflect parasympathetic and sympathetic activities of the subject's autonomic nervous system [13]. The predominating variations are originated in the phenomenon respiratory sinus arrhythmia (RSA) [28].

In medical context, the heart rate variability (HRV) is often plotted in an interval tachogram, a discrete visualization of enumerated, consecutive IBIs. However, as signals from wearable devices tend to be affected by motion artifacts, erroneously detected, spurious peaks can misalign and distort these sequences. In contrast, the HRV interval function [13] is a function of time, expressed through equation (4), that is less and, in case of artifacts, only locally disturbed.

Due to their rich information content, features derived from HRV are often used in biometric identification and authentication approaches [29], [30]. The location independence and noise immunity in combination with a high degree of intrinsic variation and uniqueness allows the use of the HRV similar to a temporal fingerprint. This specificity enables PulSync to align the time series obtained from independent devices.

\section{SynChronization Method}

PulSync utilizes the natural, irregular rhythm of the heartbeat that is ubiquitously and simultaneously available throughout the entire body surface of every living human. The ECG signal's prominent $\mathrm{R}$ peaks are significant fiducial points and serve as coincident landmarks for the alignment. In contrast to the commonly used motion signals, such as synchronization actions in acceleration signals [6], the vital sign is continuously available. Even at rest, the heart rate results in an update rate of about $0.8 \mathrm{~Hz}(48 \mathrm{bpm})$, but it can vary within the extreme boundaries ranging from $0.5 \mathrm{~Hz}$ in case of bradycardia up to $3.0 \mathrm{~Hz}$ in case of tachycardia or heavy physical activity (30-180 bpm) [31], [32]. Because the heart rate is modulated by physiological processes, the heart rate variability (HRV) interval function [13] is highly varying and unique like a fingerprint, but also location-independent and hence identical throughout the entire body surface. This singularity enables to unambiguously align the independent recordings with their local time bases by matching these HRV fingerprints.

The method's processing pipeline is illustrated in Figure 1. The heartbeat's fiducial $\mathrm{R}$ points in real time $t^{*}$ define a unique sequence of timestamps $t_{i}^{*} \in T^{*}$, allocated by equation (1). 


$$
T^{*}:=\left\langle t_{0}^{*}, \ldots, t_{i}^{*}, \ldots, t_{\infty}^{*}\right\rangle_{i \in \mathbb{N}}
$$

These heartbeat events $t_{i}^{*}$ are now captured by two exemplary devices, denoted as $A$ and $B$, which sample the individual subsets $T_{A}^{*} \subset T^{*}$ and $T_{B}^{*} \subset T^{*}$, and hence translate $t_{i}^{*}$ into their local times $t_{A}\left(t_{i}^{*}\right)$ and $t_{B}\left(t_{i}^{*}\right)$. These images $T_{A}^{*} \rightarrow T_{A}$ and $T_{B}^{*} \rightarrow T_{B}$ are specified by the equations (2) and (3).

$$
\begin{aligned}
T_{A}\left(T_{A}^{*}\right) & :=\left\langle t_{0}^{A}, \ldots, t_{j}^{A}, \ldots \mid t_{j}^{A}=t_{A}\left(t_{i}^{*}\right)\right\rangle_{j \in \mathbb{N}} \\
T_{B}\left(T_{B}^{*}\right) & :=\left\langle t_{0}^{B}, \ldots, t_{k}^{B}, \ldots \mid t_{k}^{B}=t_{B}\left(t_{i}^{*}\right)\right\rangle_{k \in \mathbb{N}}
\end{aligned}
$$

Because the devices were supposedly not started at the exact same moment, $\forall x \in \mathbb{N}: t_{x}^{A} \neq t_{x}^{B}$ applies. Furthermore, as the individual $t_{i}^{*}$ are not retrievable, the relative but unique inter-beat distances (IBI) $t_{i}^{*}-t_{i-1}^{*}$ are used as a fingerprint pattern $T_{W}^{*}=\left\langle t_{x}^{*}, \ldots, t_{x+w-1}^{*}\right\rangle$, with begin $x$ and length $w$. Because $T_{W}^{*}$ is contained in both overlapping sequences $T_{W}^{*} \subset\left(T_{A}^{*} \cap T_{B}^{*}\right)$, the sequence $T_{W}^{*} \subset T_{A}^{*}$ is translated to $t_{A}$ and used as a search window $T_{W} \subset T_{A}$ in counterpart $T_{B}$.

To enable this search by means of an approved tool, namely the cross-correlation, the sequences are first transformed into HRV interval functions by applying equation (4).

$$
\operatorname{HRV}\left(T_{X}\right):=\left\langle\left\{t_{x}, t_{x}-t_{x-1}\right\}, \ldots\right\rangle_{x=1}^{\left\|T_{X}\right\|-1}
$$

As these are sampled by means of the irregular heartbeat, the sequences have to be linearly interpolated and resampled at the sampling rate $f_{h r v}$ [13], resulting in $\alpha=\operatorname{intp}\left(H R V\left(T_{A}\right)\right)$ and $\beta=\operatorname{intp}\left(H R V\left(T_{B}\right)\right)$, for the devices $A$ and $B$ respectively.

To finally align $\alpha=\left\langle a_{i}\right\rangle_{i=1}^{\left\|T_{A}\right\|-1}$ and $\beta=\left\langle b_{i}\right\rangle_{i=1}^{\left\|T_{B}\right\|-1}$, just like the associated $T_{A}$ and $T_{B}$, a windowed segment $\alpha_{p} \subseteq \alpha$ with position $p$ is sliding along $\beta_{q} \subset \beta$ at position $q$. In doing so, a normalized cross-correlation with Pearson productmoment correlation (PPMC) coefficient $r$ [33] is applied, as defined in equation (5), to determine the position $\hat{q}$ of maximum accordance $\operatorname{argmax}_{q}\left(r\left(\alpha_{p}, \beta_{q}\right)\right)$ between the segments.

$$
\begin{aligned}
r\left(\alpha_{p}, \beta_{q}\right) & :=\frac{\operatorname{Cov}\left(\alpha_{p}, \beta_{q}\right)}{\sigma_{\alpha_{p}} \sigma_{\beta_{q}}} \\
& =\frac{\sum_{i=1}^{w}\left(a_{i}-\bar{a}\right)\left(b_{i}-\bar{b}\right)}{\sqrt{\sum_{i=1}^{w}\left(a_{i}-\bar{a}\right)^{2} \sum_{i=1}^{w}\left(b_{i}-\bar{b}\right)^{2}}}
\end{aligned}
$$

The resulting difference $p-\hat{q}$, based on $f_{h r v}$, is then multiplied by the factor $f_{s} / f_{h r v}$ to translate it back into the original time base of $f_{s}$. The original time series can finally be aligned according to the determined relative offset $\Delta=\frac{f_{s}}{f_{h r v}}(p-\hat{q})$, by adjusting their time bases towards each other.

\section{EVALUATION}

The performance of PulSync is evaluated using a publicly available dataset from 25 subjects, monitored with two independent ECG devices attached to the chest. The evaluation primarily aims at the demonstration of the general feasibility of the data-driven alignment, solely utilizing the HRV interval function as a unique fingerprint. Furthermore, the accuracy of the time series' alignment is determined by means of a metric based on the distance between their proximate peaks.

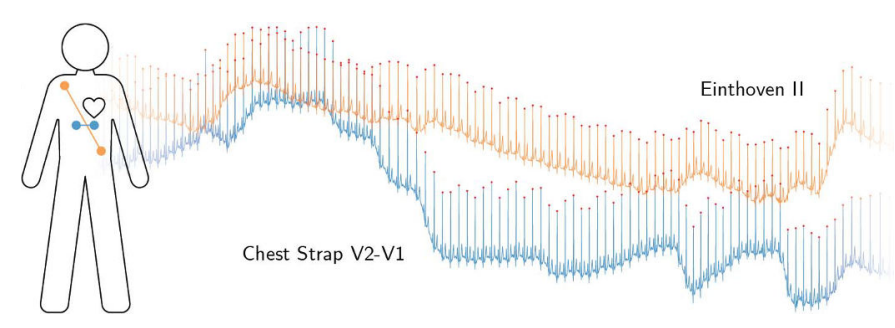

Fig. 2: The measurement setup (left) uses an exercise chest strap at the torso, resembling a V2-V1 ECG lead (blue), and the traditional Einthoven II ECG lead (orange). At the right, an excerpt from the "sitting" recordings of subject 23 [15] shows non-stationary physiological signals, superimposed by strong baseline wandering; V2-V1 lead (blue), II lead (orange); identified R peaks (red); matched by the PulSync method.

\section{A. Dataset}

The evaluation of PulSync requires recordings from different measurement sites with the largest possible diversity. The final decision was made on the research dataset 716 of Howell and Porr from the University of Glasgow which is accessible through the university's research data portal [15]. The dataset contains a large collection of two-minute ECG recordings from 25 subjects. Those performed 5 different tasks of which the "sitting" subtask has been chosen due to the availability of precise peak labels. In addition, the absence of motion artifacts in these recordings supports the evidence of the general feasibility independently from specific situations. As illustrated in Figure 2, two independent devices recorded the ECG signals at a sampling rate $f_{s}$ of $250 \mathrm{~Hz}$, pretended synchronously, with one device measuring the traditional Einthoven II lead while the second one, an exercise chest strap ECG, resembled approximately a V2-V1 lead. The dataset provides validated R peak annotations with a very high precision of \pm 1 sample. As no $\mathrm{R}$ peaks have to be identified before the derivation of the HRV interval functions, the evaluation is independent from the selection of any preprocessing and QRS detection algorithm which in turn ensures the results' reproducibility.

To enable the PPMC normalized cross-correlation, the HRV interval functions have been linearly interpolated and regularly resampled at $f_{h r v}$ of $25 \mathrm{~Hz}$. The evaluation has been conducted with a window length $w$ of $60 \mathrm{~s}$ and an overlap of $1 \%$, resulting in 101 segments of the V2-V1 lead's HRV that were shifted along and correlated with the reference HRV interval function of the Einthoven II lead. Due to boundary effects of datasets with large displacement, the first or last few segments' $r$ values were rejected to prevent a falsification of the results.

\section{B. Ground Truth}

There exist no publicly available datasets of two or more independent and at the same time hardware-synchronized ECG devices. Hence, a metric has been developed according to the fundamental assumption that ECG signals are immediately and simultaneously detectable throughout the entire body surface, and delays of the signal can be neglected. In accordance with 
the previous assumption, equation (6) introduces the alignment error $\varepsilon(j, k)$. Applied to all proximate peaks, with a minimum temporal distance, it serves as a metric of synchronicity.

$$
\varepsilon(j, k):=t_{j}^{A}-t_{k}^{B}
$$

Any displacement of the time series results in an asynchronous interference pattern that generates an $\varepsilon \neq 0$, with a large standard deviation in presence of drift. In contrast, ideally aligned time series result in a minimum $\varepsilon \rightarrow 0$, and a minimum deviation as the peaks are simultaneously following the same IBI pattern, respectively heartbeat rhythm.

\section{Limitations}

As no QRS detection has to be applied before deriving the HRV interval functions, the evaluation is independent from the selection of an algorithm, hence ensuring the reproducibility.

The previously made assumption, that the fiducial $\mathrm{R}$ points are immediately and simultaneously detectable at every arbitrary location, is exclusively valid for ECG-related measurements and the targeted accuracy in the order of a few ms. As the electrical signals propagate in tissue with a lowerbound velocity of at least $250 \mathrm{~m} \mathrm{~s}^{-1}$ [14], there are, however, perceivable delays. Assuming a maximum distance of $1.5 \mathrm{~m}$ between the measurement location on the body surface and the heart, the origin of the electrical action potentials, would consequently result in a worst-case delay of $6 \mathrm{~ms}$. This systematic bias adds to the accuracy determined, but still results in a considerably better accuracy than the dataset's original, manual alignment, and is furthermore sufficient for the targeted applications such as activity recognition [6].

\section{Results and Discussion}

Figure 3 shows a typical trace of the determined offsets $\Delta$ for 100 matched segments $\alpha_{p}$ along the reference $\beta_{q}$. Only 1 of 101 determined $r$ values is rejected due to boundary effects. The quantization steps, intersecting the straight line, indicate a small drift of about 2 samples over the entire $120 \mathrm{~s}$ length.

Figure 4 details the achieved accuracies for all 25 individual recordings while the overall results are summarized in Figure 5. Since the original recordings have been aligned manually [15], the time bases are not ideally synchronized. This is reflected by the original dataset's large initial error $\bar{\varepsilon}$ of $-28.921 \pm 89.015$ samples $(-115.684 \pm 356.060 \mathrm{~ms})$

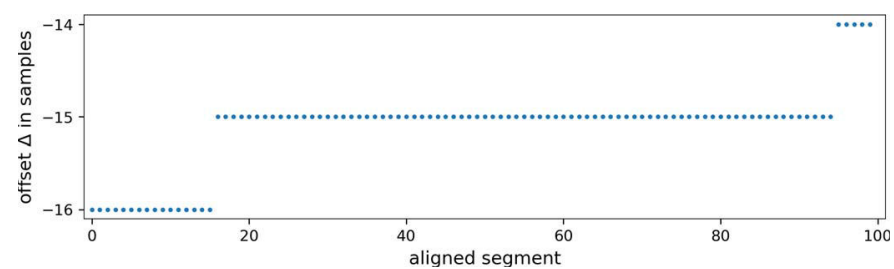

Fig. 3: Illustration of the determined offset $\Delta$ for subject 1, from 100 matched segments, 1 of 101 rejected. Visible quantization steps demonstrate the approach's sensitivity and hence the potential to detect even small drift across devices' times. PPMC $\bar{r}$ of $0.999428 \pm 0.000159, \Delta$ in samples at $250 \mathrm{~Hz}$.

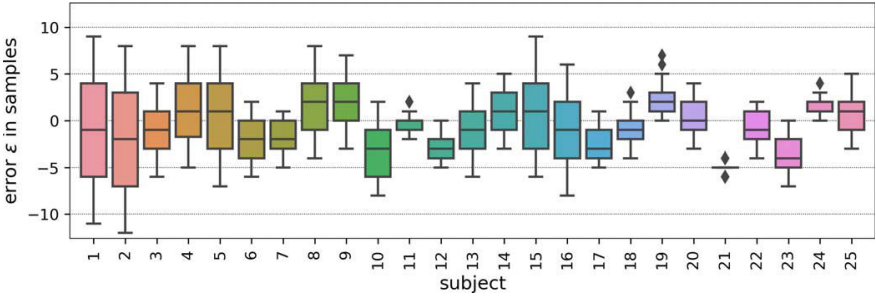

Fig. 4: Illustration of the remaining alignment error $\varepsilon$ for all individual 25 subjects' recordings. Differences between proximate peaks after the alignment, $\varepsilon$ in samples at $250 \mathrm{~Hz}$.

for the proximate peaks with a minimum distance (middle, orange). Accordingly, the misalignment (left, red), determined by means of the presented method, range from $0.035 \mathrm{~s}$ (subject 5) to even $4.961 \mathrm{~s}$ (subject 19) with an overall average $\bar{\varepsilon}$ of $15.328 \pm 428.023$ samples $(0.061 \pm 1.712 \mathrm{~s})$. In contrast, the proposed alignment approach resulted in a remaining average error $\bar{\varepsilon}$ of $-0.714 \pm 3.440$ samples $(-2.856 \pm 11.427 \mathrm{~ms})$, Besides small drift, another reason for the large standard deviation might be the HRV interval functions' coarse temporal resolution, $f_{h r v}$ of $25 \mathrm{~Hz}$, to limit the computational efforts.

Without exception, for all recordings and valid segments, Pearson's $r$ is virtually 1 and the deviation negligible as the two devices' HRV interval functions are almost identical. For signals interfered by motion artifacts, this ideal value will likely decrease according to the ratio of the affected heartbeats.

\section{CONCLUSION}

We presented PulSync, a data-driven method for the alignment of independent time bases across multiple wearable devices, which exploits the local heartbeat measurements of the common user. We propose to utilize the derived heart rate variability (HRV) interval functions as unique and locationindependent temporal fingerprints that enable the distinct and accurate matching of sensor data from body-worn devices. Our evaluation results demonstrate not only the approach's general feasibility, but also show a promising accuracy of $-0.714 \pm 3.440$ samples and accordingly $-2.856 \pm 11.427 \mathrm{~ms}$ at $250 \mathrm{~Hz}$ sampling rate. The achieved accuracy is superior to a manual alignment and is located between online, wireless synchronization techniques in the order of $\mu \mathrm{s}$ to few $\mathrm{ms}$ [3]-[5] and the offline, motion-based methods in the order of tens to hundreds of ms [2], [6]-[10]. Our evaluation revealed the approach's potential to sensitively track the relative drift between devices' local time bases. The method is particularly promising as an accurate and energy-efficient method for wearables that contain ECG, as it can be applied exclusively on recorded data and then neither requires an extra channel nor imposes an overhead on radio communication. Especially scenarios in which body-worn devices are deployed over long term, and which avoid online synchronization methods due to energy requirements, will benefit from our PulSync method.

PulSync is implemented as a Python toolbox for the offline alignment of ECG recordings. It is available for public download on this website: https://github.com/fwolling/PulSync 

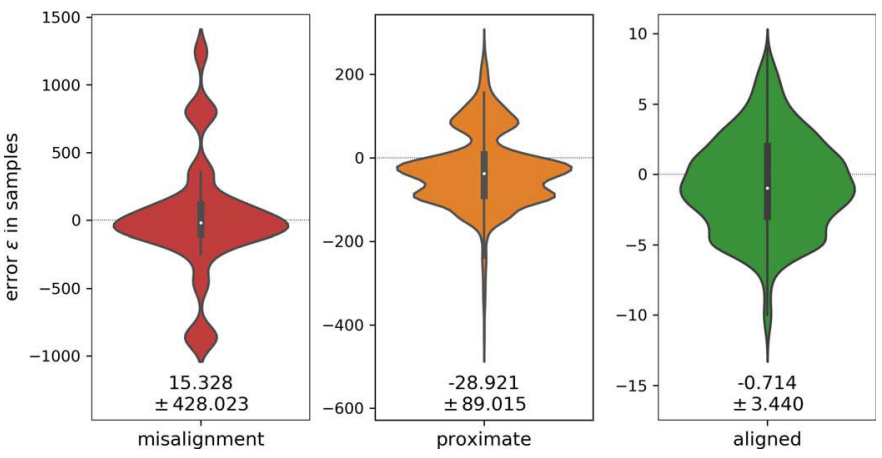

Fig. 5: Summary of the overall evaluation results. $\mathrm{Y}$ axes: error $\varepsilon$ distribution in samples at $250 \mathrm{~Hz}$. Left (red): $\varepsilon$ of assigned peaks shows initial misalignment of the original data. Middle (orange): $\varepsilon$ of interference between proximate peaks in original, unaligned data. Right (green): $\varepsilon$ of proximate peaks after the alignment. Note the different scaling of the y axes.

\section{ACKNOWLEDGMENTS}

The main author's research visit at the Biomimetics and Intelligent Systems Group of the University of Oulu, Finland has been supported by the University of Siegen and the German Academic Exchange Service (DAAD). The data has been processed by the HoRUS cluster, University of Siegen.

\section{REFERENCES}

[1] R. Ohmura, F. Naya, H. Noma, and K. Kogure, "B-pack: A bluetoothbased wearable sensing device for nursing activity recognition," in Wireless Pervasive Computing (ISWPC). IEEE, 2006, pp. 1-6.

[2] C. Wang, Z. Sarsenbayeva, C. Luo, J. Goncalves, and V. Kostakos, "Improving wearable sensor data quality using context markers," ser. UbiComp/ISWC '19. ACM, 2019.

[3] M. Ringwald and K. Romer, "Practical time synchronization for bluetooth scatternets," in 4th International Conference on Broadband Communications, Networks \& Systems, 2007, pp. 337-345.

[4] M. Pflugradt, I. Fritzsch, S. Mann et al., "A novel pulseoximeter for bluetooth synchronized measurements in a body sensor network," in EDERC'14. IEEE, 2014, pp. 21-25.

[5] H. J. G. Roberto Casas, "Synchronization in wireless sensor networks using bluetooth," in 3rd International Workshop on Intelligent Solutions in Embedded Systems. IEEE, 2005, pp. 79-88.

[6] D. Bannach, O. Amft, and P. Lukowicz, "Automatic event-based synchronization of multimodal data streams from wearable and ambient sensors," ser. EuroSSC'09. Springer, 2009, pp. 135-148.

[7] T. R. Bennett, N. Gans, and R. Jafari, "Multi-sensor data-driven synchronization using wearable sensors," ser. ISWC'15. ACM, 2015.

[8] _ "A data-driven synchronization technique for cyber-physical systems," in 2nd International Workshop on the Swarm at the Edge of the Cloud. ACM, 2015, pp. 49-54.

[9] A. Hölzemann, H. Odoemelem, and K. Van Laerhoven, "Using an in-ear wearable to annotate activity data across multiple inertial sensors," ser. EarComp' 19. ACM, 2019.

[10] T. Ahmed, M. Y. Ahmed, M. M. Rahman, E. Nemati, B. Islam, K. Vatanparvar, V. Nathan, D. McCaffrey, J. Kuang, and J. A. Gao, "Automated time synchronization of cough events from multimodal sensors in mobile devices," in International Conference on Multimodal Interaction. ACM, 2020, pp. 614-619.

[11] Y.-L. Zheng, X.-R. Ding, C. C. Y. Poon, B. P. L. Lo, H. Zhang, X.L. Zhou, G.-Z. Yang, N. Zhao, and Y.-T. Zhang, "Unobtrusive sensing and wearable devices for health informatics," IEEE transactions on biomedical engineering, vol. 61, no. 5, pp. 1538-1554, 2014.

[12] A. K. Yetisen, J. L. Martinez-Hurtado, B. Ünal, A. Khademhosseini, and H. Butt, "Wearables in medicine," Advanced Materials, vol. 30, no. 33, p. $1706910,2018$.
[13] G. Baselli, S. Cerutti, S. Civardi, F. Lombardi, A. Malliani, M. Merri, M. Pagani, and G. Rizzo, "Heart rate variability signal processing: A quantitative approach as an aid to diagnosis in cardiovascular pathologies," International Journal of Bio-Medical Computing, vol. 20, no. 1 , pp. 51-70, 1987.

[14] T. Buchner and J. Gierałtowski, "How fast does the ecg signal propagate within the body," Working Group for Cardiovascular Physics. Faculty of Physics, Warsaw University of Technology. 6th Cardiology Meets Physics \& Mathematics., vol. 6, 2015.

[15] L. Howell and B. Porr, "High precision ecg database with annotated $r$ peaks, recorded and filmed under realistic conditions." [Online]. Available: http://researchdata.gla.ac.uk/716/

[16] B. Sundararaman, U. Buy, and A. D. Kshemkalyani, "Clock synchronization for wireless sensor networks: a survey," Ad Hoc Networks, vol. 3, no. 3, pp. 281-323, 2005.

[17] S. M. Lasassmeh and J. M. Conrad, "Time synchronization in wireless sensor networks: A survey," in SoutheastCon. IEEE, 2010, pp. 242-245.

[18] H. Li and J. Tan, "Heartbeat-driven medium-access control for body sensor networks," IEEE EMBC'10, vol. 14, no. 1, pp. 44-51, 2010.

[19] B.-U. Köhler, C. Hennig, and R. Orglmeister, "The principles of software qrs detection," IEEE Engineering in Medicine and Biology Magazine, vol. 21, no. 1, pp. 42-57, 2002.

[20] D. Chabot, M. Bayer, and A. de Roos, "Instantaneous heart rates and other techniques introducing errors in the calculation of heart rate," Canadian Journal of Zoology, vol. 69, no. 4, pp. 1117-1120, 1991.

[21] R. F. Yazicioglu, T. Torfs, J. Penders, I. Romero, H. Kim, P. Merken, B. Gyselinckx, H. J. Yoo, and C. van Hoof, "Ultra-low-power wearable biopotential sensor nodes," IEEE EMBC'09, pp. 3205-3208, 2009.

[22] Y. Sun and X. B. Yu, "Capacitive biopotential measurement for electrophysiological signal acquisition: A review," IEEE Sensors Journal, vol. 16, no. 9, pp. 2832-2853, 2016.

[23] S. Ha, C. Kim, Y. M. Chi, and G. Cauwenberghs, "Low-power integrated circuit design for wearable biopotential sensing," in Wearable sensors. Elsevier, 2014, pp. 323-352.

[24] Y. M. Chi, C. Maier, and G. Cauwenberghs, "Ultra-high input impedance, low noise integrated amplifier for noncontact biopotential sensing," IEEE Journal on Emerging and Selected Topics in Circuits and Systems, vol. 1, no. 4, pp. 526-535, 2011.

[25] D. Castaneda, A. Esparza, M. Ghamari, C. Soltanpur, and H. Nazeran, "A review on wearable photoplethysmography sensors and their potential future applications in health care," International journal of biosensors \& bioelectronics, vol. 4, no. 4, pp. 195-202, 2018.

[26] F.U.S. Mattace-Raso, A. Hofman, G.C. Verwoert et al., "Determinants of pulse wave velocity in healthy people and in the presence of cardiovascular risk factors: "establishing normal and reference values'," European Heart Journal, vol. 31, no. 19, pp. 2338-2350, 2010

[27] P. H. Charlton, D. A. Birrenkott, T. Bonnici, M. A. F. Pimentel, A. E. W. Johnson, J. Alastruey, L. Tarassenko, P. J. Watkinson, R. Beale, and D. A. Clifton, "Breathing rate estimation from the electrocardiogram and photoplethysmogram: A review," IEEE Reviews in Biomedical Engineering, vol. 11, pp. 2-20, 2018.

[28] G. B. Moody, R. G. Mark, A. Zoccola, and S. Mantero, "Derivation of respiratory signals from multi-lead ecgs," vol. 12, pp. 113-116, 1985.

[29] S. A. Israel, J. M. Irvine, A. Cheng, M. D. Wiederhold, and B. K. Wiederhold, "Ecg to identify individuals," Pattern Recognition, vol. 38, no. 1, pp. 133-142, 2005.

[30] N. Akhter, S. Tharewal, V. Kale, A. Bhalerao, and K. V. Kale, "Heartbased biometrics and possible use of heart rate variability in biometric recognition systems," in Advanced computing and systems for security, ser. Advances in intelligent systems and computing. Springer, 2015, vol. 1 , pp. $15-29$.

[31] P. Dehkordi, A. Garde, B. Molavi, J. M. Ansermino, and G. A. Dumont, "Extracting instantaneous respiratory rate from multiple photoplethysmogram respiratory-induced variations," Frontiers in physiology, vol. 9, p. $948,2018$.

[32] S. Fleming, M. Thompson, R. Stevens, C. Heneghan, A. Plüddemann, I. Maconochie, L. Tarassenko, and D. Mant, "Normal ranges of heart rate and respiratory rate in children from birth to 18 years of age: a systematic review of observational studies," The Lancet, vol. 377, no. 9770, pp. 1011-1018, 2011.

[33] K. Pearson, "Note on regression and inheritance in the case of two parents," Proceedings of the Royal Society of London, vol. 58, no. 347352, pp. 240-242, 1895. 